\title{
Selection of Superior Yeast Strains for the Fermentation of Lignocellulosic Steam-Exploded Residues
}

\author{
Lorenzo Cagnin, Nicoletta Gronchi, Marina Basaglia, Lorenzo Favaro*t and \\ Sergio Casellat
}

Department of Agronomy, Food, Natural Resources, Animals and Environment (DAFNAE), University of Padova, Legnaro, Italy

The production of lignocellulosic ethanol calls for a robust fermentative yeast able to tolerate a wide range of toxic molecules that occur in the pre-treated lignocellulose. The concentration of inhibitors varies according to the composition of the lignocellulosic material and the harshness of the pre-treatment used. It follows that the versatility of the yeast should be considered when selecting a robust strain. This work aimed at the

Edited by:

Soo Rin Kim,

Kyungpook National University,

South Korea

Reviewed by:

Sujit Jagtap,

University of Illinois

at Urbana-Champaign, United States

Joana Cunha,

University of Minho, Portugal

*Correspondence:

Lorenzo Favaro

lorenzo.favaro@unipd.it

${ }^{\dagger}$ These authors have contributed equally to this work and share last

authorship

Specialty section:

This article was submitted to Microbial Physiology and Metabolism, a section of the journal

Frontiers in Microbiology

Received: 09 August 2021

Accepted: 06 October 2021

Published: 04 November 2021

Citation:

Cagnin L, Gronchi N, Basaglia M,

Favaro L and Casella S (2021)

Selection of Superior Yeast Strains for the Fermentation of Lignocellulosic

Steam-Exploded Residues.

Front. Microbiol. 12:756032.

doi: 10.3389/fmicb.2021.756032 validation of seven natural Saccharomyces cerevisiae strains, previously selected for their industrial fitness, for their application in the production of lignocellulosic bioethanol. Their inhibitor resistance and fermentative performances were compared to those of the benchmark industrial yeast S. cerevisiae Ethanol Red, currently utilized in the secondgeneration ethanol plants. The yeast strains were characterized for their tolerance using a synthetic inhibitor mixture formulated with increasing concentrations of weak acids and furans, as well as steam-exploded lignocellulosic pre-hydrolysates, generally containing the same inhibitors. The eight non-diluted liquors have been adopted to assess yeast ability to withstand bioethanol industrial conditions. The most tolerant S. cerevisiae Fm17 strain, together with the reference Ethanol Red, was evaluated for fermentative performances in two pre-hydrolysates obtained from cardoon and common reed, chosen for their large inhibitor concentrations. S. cerevisiae Fm17 outperformed the industrial strain Ethanol Red, producing up to 18 and $39 \mathrm{~g} / \mathrm{L}$ ethanol from cardoon and common reed, respectively, with ethanol yields always higher than those of the benchmark strain. This natural strain exhibits great potential to be used as superior yeast in the lignocellulosic ethanol plants.

Keywords: bioethanol, sugarcane bagasse, cardoon, common reed, industrial yeast strains, steam explosion

\section{INTRODUCTION}

Several cheap forestry and agricultural waste streams, as well as energy crops, are available for being applied as feedstocks for bioethanol production (Bhatia et al., 2017). However, such biomasses need to be pre-treated to make the cellulose more accessible to the following enzymatic hydrolysis aimed to release fermentable sugars.

Although demonstration plants using sugarcane bagasse, corn stover, wheat straw, and switchgrass are now in operation (Bhatia et al., 2017; Jansen et al., 2017), before reaching the final 
large-scale application of lignocellulosic ethanol, several challenges must be faced (Chandel et al., 2018; Dale, 2018; Liu et al., 2019) mainly about both the pre-treatment technologies and the yeast used in the processes.

Many pre-treatment technologies have been developed in the last decades and have important effects on downstream procedures, yields, and costs (da Costa Sousa et al., 2009; Nair et al., 2017; Awasthi et al., 2020; Park et al., 2020). Among the pre-treatments, steam explosion unsettles lignocellulosic materials by physical and chemical reactions, allowing a more effective subsequent enzymatic digestion. However, during steam explosion, possible inhibitors of fermentations such as phenolic compounds, furans, or weak acids are released decreasing the final ethanol yields (García et al., 2014; Morales et al., 2017).

Moreover, the fermenting yeast strains used in the secondgeneration ethanol plants, including $S$. cerevisiae Ethanol Red, have been originally selected for the application in firstgeneration ethanol distilleries. As such, there strains are generally unsuitable for the harsher conditions typical of lignocellulosic ethanol (Jönsson et al., 2013; Jansen et al., 2017; Chandel et al., 2018; Favaro et al., 2019b).

Unfortunately, while several research projects focused on the search for efficient pre-treatment technologies to maximize sugar yield (reviewed in Galbe and Zacchi, 2007; Bhutto et al., 2017; Liu et al., 2020), only a limited number approached yeast strains selection on the basis of their fermentative performances, innate resistance, and industrial fitness (Basso et al., 2008; Albers and Larsson, 2009; Pereira et al., 2011, 2014; Favaro et al., 2013a; Dubey et al., 2016; Kim et al., 2017; de Witt et al., 2019). This is a big gap of knowledge in order to optimize both substrate conversion and energy efficiency of lignocellulosic ethanol (Chandel et al., 2018).

First-generation ethanol experiences demonstrated that the efficient conversion of the raw material (corn or sugarcane) into alcohol is crucial for process economy: bioethanol industry should aim for at least $90 \%$ of theoretical yields (Walker and Walker, 2018) and even an increase of $1 \%$ would result in a considerable increase of the profit (Della-Bianca et al., 2013; Dmytruk et al., 2017). The same concept has still to be transferred to ethanol production from lignocellulose where the ethanol yields are below the industrial thresholds (Walker and Walker, 2018; Favaro et al., 2019b). Thus, the search of a vigorous yeast strain able to efficiently ferment in such industrial conditions is essential in a lignocellulosic ethanol context.

Conventional screenings for naturally tolerant S. cerevisiae strains were usually directed to individual stressors (GarayArroyo et al., 2004; Abdel-Banat et al., 2010; Mertens et al., 2018; Cunha et al., 2019). However, discovering and selecting strains with tolerance to multiple stresses, as well as assessing their fitness in simulated industrial conditions (co-presence of inhibitors, $\mathrm{pH}$ decrease, high osmolarity), would be a more realistic approach toward the development of the second-generation bioethanol industry as well reported in recent literature (Mertens et al., 2018; Brandt et al., 2019; Cunha et al., 2019; de Witt et al., 2019; Huang et al., 2019; Park et al., 2020; van Dijk et al., 2020).

In the present research paper, sugarcane bagasse, common reed, and cardoon were considered due to their potential as a source of renewable energy and their sustainability in fermentation to fuel route (Cotana et al., 2015a,b; Espada et al., 2021). Eight undiluted inhibitor-rich liquors, obtained after the steam explosion of the feedstocks mentioned above, were here used as such for both strain inhibitor tolerance assessment and fermentation to ethanol. The industrial fitness of seven selected $S$. cerevisiae strains, previously described for their high thermo- and inhibitor-tolerance (Favaro et al., 2013a, 2014; Jansen et al., 2018), was evaluated at lab scale and compared to that of the industrial reference S. cerevisiae Ethanol Red, one of the most used strain in the lignocellulose-to-ethanol processes (Dmytruk et al., 2017; Walker and Walker, 2018). The use of undetoxified steam-exploded liquors was useful to simulate the industrial environment as closely as possible.

The natural yeast strain showing the most promising inhibitor tolerance in many of the screened pre-hydrolysates, together with the reference $S$. cerevisiae Ethanol Red, were further adopted for the fermentation of two pre-hydrolysates, chosen for their high inhibitor concentration. These liquors, deriving from cardoon and common reed, were also supplemented up to 40 and $92 \mathrm{~g} / \mathrm{L}$ of glucose, respectively, to simulate the highest glucose concentration obtained by enzymatic saccharification of each steam-exploded water insoluble solid (WIS; Cotana et al., 2015a,b; Cavalaglio et al., 2016).

\section{MATERIALS AND METHODS}

\section{Feedstocks and Chemicals}

In order to obtain steam-exploded liquors with high inhibitor concentrations, samples of Phragmites australis (common reed), Cynara cardunculus (cardoon), and Saccharum officinarum (sugarcane) bagasse pre-treated by applying different conditions (residence time and temperature) resulting in increasing severity factors $\left(\log R_{0}\right)$ were investigated in this study (Table 1$) . \log R_{0^{-}}$ values (Overend and Chornet, 1987; Espirito Santo et al., 2020) were obtained according to Equation [1]:

$$
R_{0}=t e^{[(T-100) / 14.75]}
$$

where $\mathrm{t}$ is the residence time ( $\mathrm{sec} / \mathrm{min})$ and $\mathrm{T}$ is the temperature $\left({ }^{\circ} \mathrm{C}\right)$.

Briefly, pre-treatment liquors of cardoon and common reed were obtained by steam explosion. For every $\log R_{0}$-value, six consecutive explosions were executed using $500 \mathrm{~g}$ of dry biomass for each explosion. Liquors were then separated from the WIS fraction using a stainless-steel filter with a cutoff of $1 \mathrm{~mm}$ (Cotana et al., 2015a,b; Cagnin et al., 2018).

Sugarcane pre-hydrolysate was obtained in a steam explosion plant composed of a 19 L reactor, a collection tank, and a 40bar electrical boiler. Milled sugarcane bagasse samples, dried in a drying chamber to a final moisture content of $10 \%(w / w)$, were loaded into the reactor and treated for $10 \mathrm{~min}$ at $200^{\circ} \mathrm{C}$. The pre-hydrolysate was then removed using a locally manufactured dead-end press. All the pre-hydrolysates were refrigerated until use. The inhibitor and sugar contents are reported in Table $\mathbf{1 .}$ 
TABLE 1 | Pre-treatment parameters, pH, and composition of the pre-hydrolysates used in this study.

\begin{tabular}{|c|c|c|c|c|c|c|c|c|c|c|c|}
\hline \multirow[b]{2}{*}{ Substrates } & \multirow[b]{2}{*}{ Name } & \multirow[b]{2}{*}{$\log R_{0}$} & \multirow[b]{2}{*}{$\mathrm{pH}$} & \multicolumn{8}{|c|}{$g / L$} \\
\hline & & & & Glucose & Arabinose & Xylose & Formic acid & Acetic acid & Levulinic acid & Furfural & HMF \\
\hline \multirow[t]{3}{*}{ P. australis } & Pa1 & 3.60 & 3.75 & $0.14 \pm 0.01$ & $0.25 \pm 0.02$ & $1.24 \pm 0.11$ & $0.32 \pm 0.01$ & $1.00 \pm 0.08$ & n.d. & $0.24 \pm 0.01$ & $0.05 \pm 0.01$ \\
\hline & $\mathrm{Pa} 2$ & 4.00 & 3.29 & $0.29 \pm 0.02$ & $0.35 \pm 0.01$ & $2.04 \pm 0.18$ & $0.78 \pm 0.05$ & $2.18 \pm 0.11$ & $0.001 \pm 0.001$ & $0.97 \pm 0.07$ & $0.13 \pm 0.01$ \\
\hline & Pa3 & 4.40 & 3.23 & $0.43 \pm 0.02$ & $0.09 \pm 0.01$ & $0.53 \pm 0.04$ & $1.28 \pm 0.11$ & $3.50 \pm 0.25$ & $0.008 \pm 0.001$ & $1.43 \pm 0.11$ & $0.48 \pm 0.03$ \\
\hline \multirow[t]{4}{*}{ C. cardunculus } & Cc1 & 3.85 & 4.10 & $0.02 \pm 0.01$ & $0.01 \pm 0.01$ & $0.23 \pm 0.01$ & $0.50 \pm 0.03$ & $0.71 \pm 0.06$ & $0.002 \pm 0.001$ & $0.09 \pm 0.01$ & $0.05 \pm 0.01$ \\
\hline & Cc2 & 4.02 & 3.96 & $0.30 \pm 0.02$ & $0.11 \pm 0.02$ & $2.15 \pm 0.12$ & $1.73 \pm 0.11$ & $2.15 \pm 0.11$ & $0.003 \pm 0.001$ & $0.36 \pm 0.02$ & $0.20 \pm 0.02$ \\
\hline & Cc3 & 4.28 & 3.83 & $0.20 \pm 0.02$ & $0.20 \pm 0.02$ & $2.20 \pm 0.17$ & $2.18 \pm 0.15$ & $2.76 \pm 0.24$ & $0.004 \pm 0.001$ & $0.44 \pm 0.02$ & $0.28 \pm 0.02$ \\
\hline & Cc4 & 4.53 & 3.49 & $0.13 \pm 0.01$ & $0.03 \pm 0.01$ & $1.91 \pm 0.11$ & $4.28 \pm 0.28$ & $5.80 \pm 0.41$ & $0.011 \pm 0.003$ & $0.64 \pm 0.04$ & $0.39 \pm 0.02$ \\
\hline S. officinarum & So1 & 4.65 & 3.28 & $0.50 \pm 0.03$ & $0.40 \pm 0.03$ & $2.95 \pm 0.18$ & $3.00 \pm 0.19$ & $11.20 \pm 0.90$ & $0.019 \pm 0.005$ & $1.70 \pm 0.12$ & $0.50 \pm 0.03$ \\
\hline
\end{tabular}

Severity factor LogRo correlates with the harshness of the pre-treatment (Cotana et al., 2015a,b). n.d., not detected.

All chemicals, media components, and supplements were of analytical grade standard.

\section{Yeast Strains}

The phenotypes and sources of the S. cerevisiae strains used in this work are summarized in Table 2. Yeast strains pre-cultures were grown in YPD medium (g/L: yeast extract, 10; peptone, 20; glucose, 20) at $30^{\circ} \mathrm{C}$ on a rotary shaker set at $130 \mathrm{rpm}$ unless otherwise stated.

\section{Evaluation of Inhibitor Tolerance of Selected Wild Type and Industrial Yeast}

Seven natural yeast strains (Fm17, Fm89, Fm90, Fm96, M2n, MEL2, and YI30) were screened for their industrial fitness using Ethanol Red as benchmark industrial yeast. Inhibitor tolerance in the presence of four synthetic inhibitor mixtures and eight inhibitor-rich lignocellulosic pre-hydrolysates was assessed.

\section{Inhibitor Tolerance in Synthetic Inhibitor Mixtures}

Yeast strains were firstly evaluated for their inhibitor tolerance in filter-sterilized $(0.22 \mu \mathrm{m})$ defined Yeast Nitrogen Base (YNB) medium supplemented with $20 \mathrm{~g} / \mathrm{L}$ of glucose and containing increasing concentrations of weak acids (acetic, formic acids) and furans (furfural, HMF: 5-hydroxymethyl-2-furaldehyde). Inhibitors were formulated into four mixtures, namely, $\mathrm{RC}_{25}$, $\mathrm{RC}_{50}, \mathrm{RC}_{100}$, and $\mathrm{RC}_{200}$ (RC: Relative Concentration) obtained by adding increasing doses of each toxic compound. $\mathrm{RC}_{100}$ was formulated using the highest concentrations of each tested inhibitor present in many lignocellulosic pre-hydrolysates, namely, acetic acid 7.20, formic acid 2.40, furfural 2.70, HMF $3.78 \mathrm{~g} / \mathrm{L}$ (Favaro et al., 2016, 2019a; Jönsson and Martín, 2016; Bhatia et al., 2017; Bhutto et al., 2017; Dmytruk et al., 2017; Jansen et al., 2018; Roscini et al., 2019). $\mathrm{RC}_{25}$ and $\mathrm{RC}_{50}$ mixtures were, respectively, obtained as 4 -fold and 2-fold dilutions of $\mathrm{RC}_{100} . \mathrm{RC}_{200}$ is a 2 -fold concentration of $\mathrm{RC}_{100}$. $\mathrm{pH}$ was adjusted to 5.0 , using $5 \mathrm{M} \mathrm{NaOH}$. This particular $\mathrm{pH}$ is widely used in the bioethanol production process (Kádár et al., 2007). The detailed inhibitor composition of each mixture is reported in Table 3.

Overnight cultures of each yeast strain, grown at $30^{\circ} \mathrm{C}$ in YNB medium containing $20 \mathrm{~g} / \mathrm{L}$ of glucose, were transferred, in biological triplicate, at an inoculum concentration of $1 \times 10^{6}$ cells $/ \mathrm{mL}$, in $2 \mathrm{~mL}$ Eppendorf tubes containing $0.9 \mathrm{~mL}$ of medium. After $40 \mathrm{~h}$ of growth $\left(30^{\circ} \mathrm{C}, 130 \mathrm{rpm}\right)$, the optical density at $600 \mathrm{~nm}\left(\mathrm{OD}_{600}\right)$ was measured. For each strain, the tolerance was estimated as relative growth (\%), calculated as the ratio between measured $\mathrm{OD}_{600}$-values of the medium with inhibitors and the control medium, devoid of any inhibitor mixture (Favaro et al., 2014).

\section{Inhibitor Tolerance in Lignocellulosic Pre-hydrolysates}

Inhibitor tolerance of the strains was also assayed in eight lignocellulosic pre-hydrolysates, obtained by steam explosion of P. australis, C. cardunculus, and S. officinarum bagasse (Table 1).

Overnight cultures of each strain were used to inoculate a volume of $200 \mu \mathrm{l}$ of each lignocellulosic hydrolysate containing YNB and $20 \mathrm{~g} / \mathrm{L}$ of glucose. $\mathrm{pH}$ of the medium was not modified. The medium was filter-sterilized through $0.22 \mu \mathrm{m}$. The experiment was carried out in quintuplicate for each condition in 96-well plates using the multimode microplate reader TECAN Spark 10 M (Tecan Group Ltd., Switzerland). An increase in $\mathrm{OD}_{600}$-value indicated the ability of the strain to sustain growth in the presence of the specific pre-hydrolysate.

Similarly, yeast strains were evaluated in $0.9 \mathrm{~mL}$ of $\mathrm{YNB}$ medium formulated with pre-hydrolysates Pa3, Cc3, Cc4, and containing $20 \mathrm{~g} / \mathrm{L}$ glucose. $\mathrm{pH}$ was either not modified or adjusted at values of 4.5 and 5.0 by adding $5 \mathrm{M} \mathrm{NaOH}$. The experiment was carried out in triplicate for each condition. Cell culture preparation, analytical methods, and evaluation of inhibitor tolerance in terms of relative growth were performed as defined in the section "Inhibitor Tolerance in Synthetic Inhibitor Mixtures."

\section{Fermentation of Lignocellulosic Pre-hydrolysates}

Fermentation performances of S. cerevisiae Fm17 and Ethanol Red were evaluated in YNB medium formulated with cardoon (Cc3) or common reed (Pa3) pre-hydrolysates supplemented with up to $20 \mathrm{~g} / \mathrm{L}$ of glucose. Moreover, the two pre-treatment liquors were supplemented with YNB and 40 or $92 \mathrm{~g} / \mathrm{L}$ of glucose, respectively, to simulate the highest glucose concentration obtained by enzymatic saccharification of each steam-exploded 
TABLE 2 | Yeast strains used in this study.

\begin{tabular}{|c|c|c|c|}
\hline Strain & $\begin{array}{l}\text { Relevant } \\
\text { phenotype }\end{array}$ & References & Source \\
\hline $\begin{array}{l}\text { S. cerevisiae } \\
\text { Ethanol Red }\end{array}$ & $\begin{array}{l}\text { Industrial strain for } \\
\text { bioethanol } \\
\text { production }\end{array}$ & $\begin{array}{l}\text { Lesaffre } \\
\text { (Marcq-en- } \\
\text { Barśul, } \\
\text { France) }\end{array}$ & Fermentis division \\
\hline $\begin{array}{l}\text { S. cerevisiae } \\
\text { Fm17 }\end{array}$ & $\begin{array}{l}\text { Newly isolated } \\
\text { strain with high } \\
\text { inhibitor tolerance }\end{array}$ & $\begin{array}{l}\text { Favaro et al., } \\
2013 a\end{array}$ & $\begin{array}{l}\text { DAFNAE collection } \\
\text { (University of Padova, } \\
\text { Italy) }\end{array}$ \\
\hline $\begin{array}{l}\text { S. cerevisiae } \\
\text { Fm89 }\end{array}$ & $\begin{array}{l}\text { Newly isolated } \\
\text { strain with high } \\
\text { inhibitor tolerance }\end{array}$ & $\begin{array}{l}\text { Favaro et al., } \\
2013 a\end{array}$ & $\begin{array}{l}\text { DAFNAE collection } \\
\text { (University of Padova, } \\
\text { Italy) }\end{array}$ \\
\hline $\begin{array}{l}\text { S. cerevisiae } \\
\text { Fm90 }\end{array}$ & $\begin{array}{l}\text { Newly isolated } \\
\text { strain with high } \\
\text { inhibitor tolerance }\end{array}$ & $\begin{array}{l}\text { Favaro et al., } \\
2013 a\end{array}$ & $\begin{array}{l}\text { DAFNAE collection } \\
\text { (University of Padova, } \\
\text { Italy) }\end{array}$ \\
\hline $\begin{array}{l}\text { S. cerevisiae } \\
\text { Fm96 }\end{array}$ & $\begin{array}{l}\text { Newly isolated } \\
\text { strain with high } \\
\text { inhibitor tolerance }\end{array}$ & $\begin{array}{l}\text { Favaro et al., } \\
2014\end{array}$ & $\begin{array}{l}\text { DAFNAE collection } \\
\text { (University of Padova, } \\
\text { Italy) }\end{array}$ \\
\hline $\begin{array}{l}\text { S. cerevisiae } \\
\text { M2n }\end{array}$ & $\begin{array}{l}\text { Industrial distillery } \\
\text { strain }\end{array}$ & $\begin{array}{l}\text { Viktor et al., } \\
2013\end{array}$ & $\begin{array}{l}\text { Stellenbosch University } \\
\text { (South Africa) }\end{array}$ \\
\hline $\begin{array}{l}\text { S. cerevisiae } \\
\text { MEL2 }\end{array}$ & $\begin{array}{l}\text { Industrial strain with } \\
\text { high fermentative } \\
\text { vigor }\end{array}$ & $\begin{array}{l}\text { Favaro et al., } \\
2013 b\end{array}$ & $\begin{array}{l}\text { DAFNAE collection } \\
\text { (University of Padova, } \\
\text { Italy) }\end{array}$ \\
\hline $\begin{array}{l}\text { S. cerevisiae } \\
\text { YI30 }\end{array}$ & $\begin{array}{l}\text { Wild type strain } \\
\text { with high inhibitor } \\
\text { tolerance }\end{array}$ & $\begin{array}{l}\text { Jansen et al., } \\
2018\end{array}$ & $\begin{array}{l}\text { Stellenbosch University } \\
\text { (South Africa) }\end{array}$ \\
\hline
\end{tabular}

TABLE 3 | Inhibitor composition of four quaternary mixtures used to assess yeast inhibitor tolerance.

\begin{tabular}{lcccc}
\hline & \multicolumn{4}{c}{ Concentration (g/L) } \\
\cline { 2 - 5 } Inhibitor & $\mathbf{R C}_{\mathbf{2 5}}$ & $\mathbf{R C}_{\mathbf{5 0}}$ & $\mathbf{R C}_{\mathbf{1 0 0}}$ & $\mathbf{R C}_{\mathbf{2 0 0}}$ \\
\hline Acetic acid & 1.80 & 3.60 & 7.20 & 14.40 \\
Formic acid & 0.60 & 1.20 & 2.40 & 4.80 \\
Furfural & 0.68 & 1.35 & 2.70 & 5.40 \\
HMF & 0.95 & 1.89 & 3.78 & 7.56 \\
\hline
\end{tabular}

Before adjustment, $\mathrm{pH}$-values of inhibitor mixtures $R C_{25}, R C_{50}, R C_{100}$, and $R C_{200}$ were $2.60,2.50,2.40$, and 2.20, respectively.

WIS (Cotana et al., 2015a,b; Cavalaglio et al., 2016). After pH was adjusted to 5.0 using $5 \mathrm{M} \mathrm{NaOH}$, broths were sterilized using a $0.22 \mu \mathrm{m}$ sterile filter.

Pre-cultures of yeast cells grown to stationary phase in YNB medium containing $20 \mathrm{~g} / \mathrm{L}$ of glucose were used to inoculate $50 \mathrm{~mL}$ medium to an initial $\mathrm{OD}_{600}$ of 1.0 in $55 \mathrm{~mL}$ glass serum bottles. The small-scale fermentations were carried out in triplicate under oxygen-limited conditions. Bottles were sealed with rubber stoppers, incubated at $30^{\circ} \mathrm{C}$, and mixed on a magnetic stirrer. Growth was measured as $\mathrm{OD}_{600}$ and samples, taken through a capped syringe needle pierced through the bottle stopper, were stored at $-20^{\circ} \mathrm{C}$. Collected samples were filtered through a $0.22 \mu \mathrm{m}$ pore filter and diluted prior to HPLC (high-performance liquid chromatography) analysis performed as described in the section "Analytical Methods, Calculations, and Statistical Analysis."

\section{Analytical Methods, Calculations, and Statistical Analysis}

Samples of lignocellulosic pre-hydrolysates and liquid fractions during small scale fermentations were analyzed for ethanol, glycerol, arabinose, galactose, glucose, xylose, mannose, sucrose, maltose, cellobiose, acetic acid, formic acid, levulinic acid, furfural, and HMF. Liquid chromatography analysis was performed using a Shimadzu Nexera HPLC system, equipped with a RID-10A refractive index detector (Shimadzu, Kyoto, Japan). The chromatographic separations were performed using a Phenomenex Rezex ROA-Organic Acid $\mathrm{H}^{+}(8 \%)$ column $(300 \mathrm{~mm} \times 7.8 \mathrm{~mm})$. The column temperature was set at $65^{\circ} \mathrm{C}$, and the analysis was performed at a flow rate of $0.6 \mathrm{~mL} / \mathrm{min}$ using isocratic elution, with $0.01 \mathrm{M} \mathrm{H}_{2} \mathrm{SO}_{4}$ as a mobile phase (Favaro et al., 2010). Analytes were identified by comparing their retention times, and the concentrations were calculated using calibration curves of the corresponding external standard.

The ethanol yield $\left(Y_{\mathrm{E} / \mathrm{G}}\right)$ from glucose was calculated as the highest amount of ethanol produced per gram of consumed glucose $(\mathrm{g} / \mathrm{g})$. The volumetric productivity $(\mathrm{Q})$ was based on grams of the highest ethanol produced per liter of culture medium per hour $\left(\mathrm{g} / \mathrm{L} \mathrm{h}^{-1}\right)$. $Q_{\max }$ was calculated as the highest volumetric productivity along the fermentations.

Statistical analyses were obtained using the Graphpad Prism 5 package (Graphpad Software, Inc., San Diego, CA, United States). Mean values, standard deviations, and descriptive statistics were calculated. Fermentations performances were analyzed by ANOVA (ANalysis Of Variance) using Duncan test post hoc means differentiation.

\section{RESULTS AND DISCUSSION \\ Screening of Saccharomyces cerevisiae Yeast Strains for Inhibitor Tolerance}

Seven S. cerevisiae strains, namely, Fm17, Fm89, Fm90, Fm96, M2n, MEL2, and YI30, were previously described for their potential in various bioethanol applications (Favaro et al., 2013a, 2014; Jansen et al., 2018). In this study, these strains were further characterized with the final aim of assessing their promise to be used for lignocellulosic ethanol production. As such, S. cerevisiae Ethanol Red was specifically chosen as reference industrial yeast (Walker and Walker, 2018; Favaro et al., 2019b).

\section{Inhibitor Tolerance in Synthetic Inhibitor Mixtures}

Inhibitor resistance was firstly evaluated in the presence of four synthetic mixtures of inhibitors most commonly found in lignocellulosic pre-hydrolysates. S. cerevisiae strains were grown in YNB medium containing $20 \mathrm{~g} / \mathrm{L}$ of glucose and increasing concentrations of synthetic inhibitors, weak acids (acetic, formic acid), and furans (furfural, HMF). As described in the section "Inhibitor Tolerance in Synthetic Inhibitor Mixtures," the tolerance of each strain was evaluated as relative growth (\%) by comparing the cell growth in the medium containing inhibitors with that lacking these compounds, after $40 \mathrm{~h}$ incubation at $30^{\circ} \mathrm{C}$ (Table 4). 
TABLE 4 | Influence of increasing concentrations of mixtures of weak acids (acetic and formic acid) and furans (furfural and HMF) on aerobic yeast growth in defined YNB medium supplemented with $20 \mathrm{~g} / \mathrm{L}$ of glucose.

\begin{tabular}{lcccccccc}
\hline \multicolumn{10}{c}{ Relative growth (\%) } \\
\cline { 2 - 8 } & Fm17 & Fm89 & Fm90 & Fm96 & M2n & MEL2 & YI30 & $\begin{array}{c}\text { Ethanol } \\
\text { Red }\end{array}$ \\
\hline $\mathrm{RC}_{25}$ & $94 \pm 4$ & $81 \pm 4$ & $87 \pm 4$ & $79 \pm 4$ & $50 \pm 4$ & $82 \pm 4$ & $85 \pm 3$ & $65 \pm 3$ \\
$\mathrm{RC}_{50}$ & $71 \pm 4$ & $62 \pm 3$ & $59 \pm 3$ & $53 \pm 3$ & $21 \pm 1$ & $60 \pm 3$ & $63 \pm 3$ & $44 \pm 2$ \\
$\mathrm{RC}_{100}$ & $60 \pm 3$ & $45 \pm 2$ & $42 \pm 2$ & $39 \pm 3$ & $14 \pm 1$ & $28 \pm 1$ & $55 \pm 2$ & $11 \pm 1$ \\
$\mathrm{RC}_{200}$ & 0 & 0 & 0 & 0 & 0 & 0 & 0 & 0
\end{tabular}

$\mathrm{pH}$ was adjusted to 5.0 with $5 \mathrm{M} \mathrm{NaOH}$. Inhibitor tolerance values are expressed as relative growth (\%) for each strain after $40 \mathrm{~h}$. Results are the means of three replicates $( \pm S D)$.

Inhibitor mixtures hindered cell growth with different degrees of severity. As expected, the relative growth decreased by increasing the concentration of inhibitors. S. cerevisiae M2n and Ethanol Red displayed the lowest tolerance already in the presence of the most diluted mixture $\left(\mathrm{RC}_{25}\right)$, with a relative growth of 50 and 65\%, respectively. Conversely, Fm17 exhibited the highest degree of tolerance to the inhibitors formulations, with values of 94,71 , and $60 \%$ in $\mathrm{RC}_{25}, \mathrm{RC}_{50}$, and $\mathrm{RC}_{100}$, respectively. A slightly lower extent of tolerance has been also measured for S. cerevisiae YI30, recently proposed as a promising strain for lignocellulosic ethanol (Jansen et al., 2018). By contrast, $\mathrm{RC}_{200}$ inhibited growth of all strains tested.

\section{Using Lignocellulosic Pre-hydrolysates to Assess Yeast Inhibitor Tolerance}

Although the synthetic mixtures were often used for assessing the inhibitor tolerance of $S$. cerevisiae strains (Martín and Jönsson, 2003; Favaro et al., 2013a, 2014; Viktor et al., 2013; Wimalasena et al., 2014; Jansen et al., 2018), the ability of yeast cells to grow and withstand real lignocellulosic pre-treated materials could greatly vary, due to the hindering action of other toxic compounds that cannot be easily identified or quantified (Chandel et al., 2018). The objective of this work was to select yeast strains for second-generation bioethanol production in the industrial context, based on their high level of robustness and strong fermentative performances. Therefore, for the first time, several pre-hydrolysates from steam pre-treated lignocellulosic materials, namely, sugarcane bagasse, common reed, and cardoon, were used as a source of inhibitors. These feedstocks, selected as model of other cheap and abundant lignocellulosic substrates, together with steam explosion, which is one of the most commonly used pre-treatments (Mussatto, 2016), would result in conditions representative for second-generation ethanol production.

In order to obtain a cluster of liquors enriched in inhibitory compounds, several $\log R_{0}$-values were applied for the steamexplosion of the lignocellulosic materials, resulting in the release of different inhibitor concentrations and small amounts of xylose, arabinose, and glucose (Table 1). The steam explosion of S. officinarum bagasse yielded the pre-hydrolysate So1, having the highest amounts of aliphatic acids (about $14.2 \mathrm{~g} / \mathrm{L}$ ) and furans $(2.2 \mathrm{~g} / \mathrm{L})$. These values agree with those described in other steam-exploded sugarcane bagasse samples (Martìn et al., 2002; Fockink et al., 2018). The higher severity, the higher release of inhibitors in both $P$. australis and $C$. cardunculus prehydrolysates (Table 1). Among the $P$. australis pre-treatments liquors, $\mathrm{Pa} 3$ was the richest in terms of inhibitors with almost 4.8 and $2.0 \mathrm{~g} / \mathrm{L}$ of weak acids and furans, respectively. On the other hand, $\mathrm{Cc} 4$ contained the uppermost levels of inhibitors among the pre-hydrolysates of C. cardunculus. Such concentrations compare well with those recently reported for cardoon and common read steam gun pre-treatments (Bułkowska and Klimiuk, 2016).

The ability of the yeast strains to grow in the presence of eight undiluted pre-hydrolysates was firstly evaluated in a qualitative high-throughput assay using YNB containing $20 \mathrm{~g} / \mathrm{L}$ of glucose, as described in the section "Inhibitor Tolerance in Lignocellulosic Pre-hydrolysates." Yeast growth was determined by detecting increased turbidity of the medium (Supplementary Table 1). All strains were able to grow in pre-hydrolysates $\mathrm{Pa} 1$ and $\mathrm{Pa} 2$ from $P$. australis and in $\mathrm{Cc} 1, \mathrm{Cc} 2$, and $\mathrm{Cc} 3$ from C. cardunculus, except for Fm89 strain in Pa2. Pre-treatment liquors $\mathrm{Pa} 3$ from P. australis, Cc4 from C. cardunculus, and Sol from S. officinarum bagasse did not support the growth of any yeast, indicating that their concentrations of toxic chemical species were higher than yeast could tolerate. This hypothesis is confirmed by the large inhibitor concentrations present in each of these prehydrolysates (Table 1). In fact, $\mathrm{Pa} 3$ contains the uppermost amount of inhibitors found in the pre-hydrolysates originating from $P$. australis and one of the strongest concentrations of furans among all the pre-hydrolysates. Similarly, for Cc4, which appears as the harshest liquor from C. cardunculus, with very high concentrations of weak acids (nearly $10 \mathrm{~g} / \mathrm{L}$ ).

With the aim to select highly tolerant yeast, $\mathrm{Pa} 3, \mathrm{Cc} 3$, and $\mathrm{Cc} 4$ liquors were chosen for additional experimental activities on yeast inhibitor resistance. Since the use of undiluted substrate would be the best criterion to adopt, So1 was excluded because

TABLE 5 | Influence of different lignocellulosic pre-hydrolysates on yeast growth in defined YNB medium supplemented with $20 \mathrm{~g} / \mathrm{L}$ of glucose with or without $\mathrm{pH}$ adjustment to $\mathrm{pH} 5.0$ with $5 \mathrm{M} \mathrm{NaOH}$.

\begin{tabular}{|c|c|c|c|c|c|c|}
\hline \multirow[b]{2}{*}{ Strain } & \multicolumn{2}{|c|}{$\mathrm{Pa} 3$} & \multicolumn{2}{|c|}{ Cc3 } & \multicolumn{2}{|c|}{ Cc4 } \\
\hline & $\begin{array}{l}\text { Unaltered } \\
\text { (pH 3.23) }\end{array}$ & $\begin{array}{l}\text { Adjusted } \\
\text { (pH 5.00) }\end{array}$ & $\begin{array}{c}\text { Unaltered } \\
\text { (pH 3.83) }\end{array}$ & $\begin{array}{l}\text { Adjusted } \\
\text { (pH 5.00) }\end{array}$ & $\begin{array}{l}\text { Unaltered } \\
\text { (pH 3.49) }\end{array}$ & $\begin{array}{l}\text { Adjusted } \\
\text { (pH 5.00) }\end{array}$ \\
\hline Fm17 & 0 & $69 \pm 4$ & $62 \pm 3$ & $88 \pm 5$ & 0 & $66 \pm 4$ \\
\hline Fm89 & 0 & $68 \pm 4$ & $48 \pm 3$ & $61 \pm 3$ & 0 & $63 \pm 3$ \\
\hline Fm90 & 0 & $61 \pm 3$ & $61 \pm 4$ & $80 \pm 4$ & 0 & $60 \pm 4$ \\
\hline Fm96 & 0 & $9 \pm 1$ & $50 \pm 3$ & $79 \pm 4$ & 0 & $54 \pm 3$ \\
\hline M2n & 0 & $16 \pm 1$ & $53 \pm 3$ & $57 \pm 3$ & 0 & $60 \pm 3$ \\
\hline MEL2 & 0 & $8 \pm 1$ & $30 \pm 2$ & $61 \pm 3$ & 0 & $56 \pm 3$ \\
\hline YI30 & 0 & $57 \pm 3$ & $70 \pm 4$ & $81 \pm 5$ & 0 & $60 \pm 3$ \\
\hline Ethanol & 0 & $37 \pm 2$ & $50 \pm 3$ & $78 \pm 4$ & 0 & $63 \pm 3$ \\
\hline
\end{tabular}

Inhibitor tolerance is expressed as relative growth (\%) measured for each strain after $40 \mathrm{~h}$ in $\mathrm{YNB}$, and results are the means of three replicates ( $\pm S D$ ). 


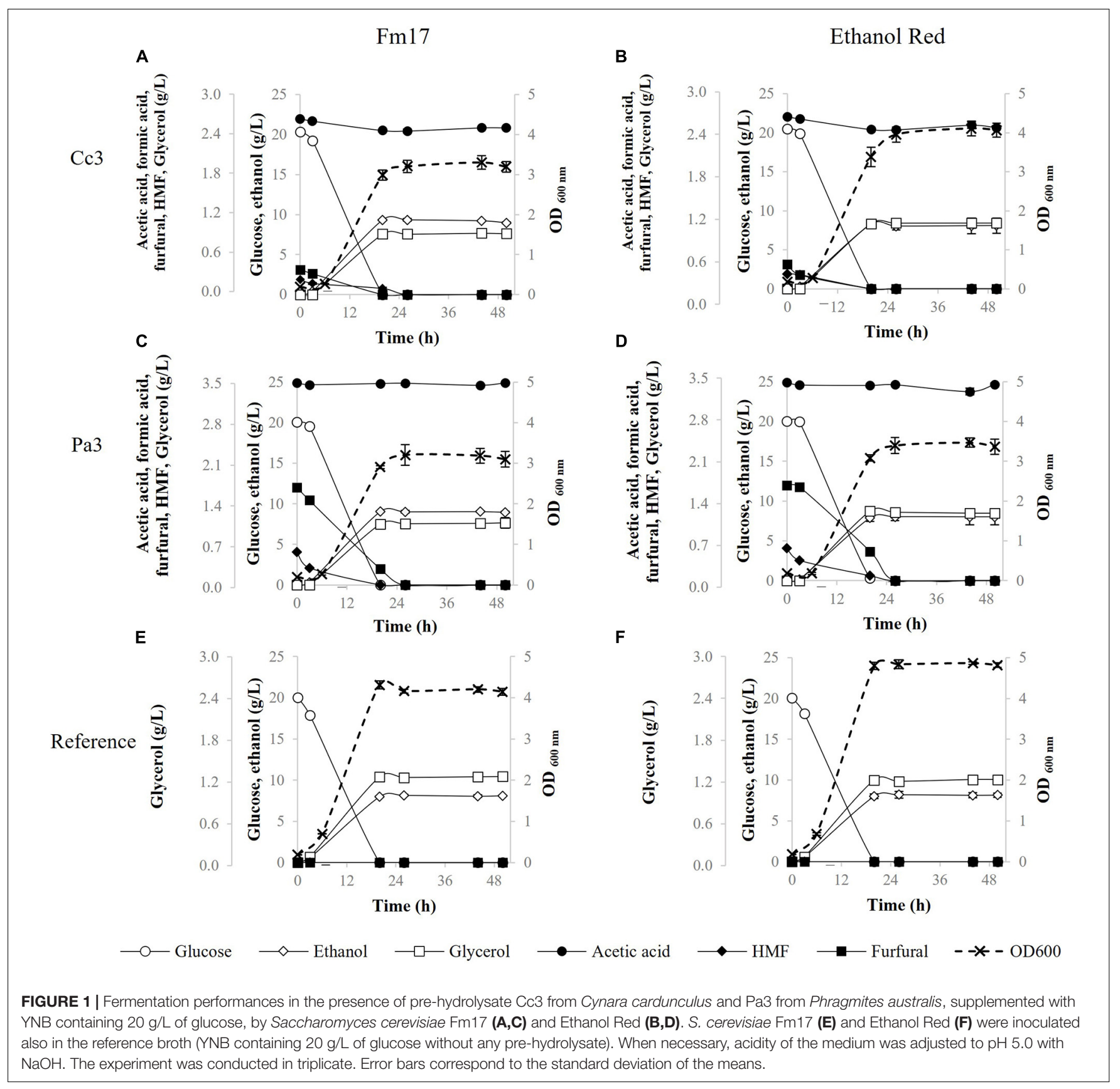

of its high inhibitor content (Table 1) exceeding the ability of the yeast to cope with (Palmqvist and Hahn-Hägerdal, 2000; Jönsson et al., 2013; Jönsson and Martín, 2016). Relative inhibitor tolerance of the eight strains was quantified in YNB medium containing $20 \mathrm{~g} / \mathrm{L}$ of glucose and formulated with prehydrolysates $\mathrm{Pa} 3, \mathrm{Cc} 3$, and $\mathrm{Cc} 4$, without altering the $\mathrm{pH}$ of the media. In these conditions, yeast growth was completely inhibited in $\mathrm{Pa} 3$ and $\mathrm{Cc} 4$, while all strains could grow in the presence of the pre-hydrolysate Cc3 (Table 5). S. cerevisiae YI30 and Fm17 exhibited the highest relative growth values, 70 and $62 \%$, respectively. The reference Ethanol Red showed lower inhibitor tolerance. Higher toxicity of pre-hydrolysates $\mathrm{Pa} 3$ and $\mathrm{Cc} 4$ is likely caused by the higher amounts of acetic acid, furfural, and HMF (Table 1), compared to the less toxic Cc3.

Overall, the selected natural yeast strains exhibited a versatility toward multiple pre-treated materials greater than the benchmark industrial yeast (Supplementary Table 1 and Table 5). This is one of the main achievements for a lignocellulosic ethanol yeast (Dmytruk et al., 2017; Walker and Walker, 2018; Favaro et al., 2019b).

The experiment was replicated after adjusting medium acidity to $\mathrm{pH} 5.0$ (Table 5). $\mathrm{pH}$ adjustment resulted in an overall improvement of relative growth: all strains did grow in the presence of pre-hydrolysates $\mathrm{Pa} 3$ and $\mathrm{Cc} 3$, as well as in Cc4. 
TABLE 6 | Fermentative performances at $30^{\circ} \mathrm{C}$ of Saccharomyces cerevisiae strain Fm17 and the benchmark S. cerevisiae Ethanol Red (ER) when incubated in the presence of pre-hydrolysate Cc3 and Pa3 supplemented with 20, 40, or $92 \mathrm{~g} / \mathrm{L}$ glucose.

\begin{tabular}{|c|c|c|c|c|c|c|c|c|c|c|c|}
\hline \multirow[t]{2}{*}{$\begin{array}{l}\text { Glucose } \\
\text { concentration (g/L) }\end{array}$} & \multirow[t]{2}{*}{$\begin{array}{c}\text { Pre- } \\
\text { hydrolysate }\end{array}$} & \multicolumn{2}{|c|}{$\begin{array}{l}\text { Highest glycerol } \\
\text { concentration (g/L) }\end{array}$} & \multicolumn{2}{|c|}{$\begin{array}{l}\text { Highest ethanol } \\
\text { concentration (g/L) }\end{array}$} & \multicolumn{2}{|c|}{$Y_{E / G}(g / g)$} & \multicolumn{2}{|c|}{$Q\left(g / L h^{-1}\right)$} & \multicolumn{2}{|c|}{$Q_{\max }\left(g / L h^{-1}\right)$} \\
\hline & & Fm17 & ER & Fm17 & ER & Fm17 & ER & Fm17 & ER & Fm17 & ER \\
\hline 20 & - & $0.79 \pm 0.03$ & $0.74 \pm 0.03$ & $8.17 \pm 0.38$ & $8.21 \pm 0.38$ & $0.41(80 \%)$ & $0.41(80 \%)$ & 0.31 & 0.32 & 0.40 & 0.40 \\
\hline 20 & Cc3 & $0.83 \pm 0.03$ & $0.74 \pm 0.04$ & $9.34 \pm 0.27$ & $8.26 \pm 0.21$ & $0.46(90 \%)$ & $0.40(79 \%)$ & 0.36 & 0.41 & 0.47 & 0.41 \\
\hline 20 & Рa3 & $0.80 \pm 0.04$ & $0.72 \pm 0.03$ & $9.04 \pm 0.28$ & $8.01 \pm 0.25$ & $0.45(88 \%)$ & 0.40 (78\%) & 0.45 & 0.31 & 0.45 & 0.39 \\
\hline 92 & - & $3.27 \pm 0.13$ & $3.27 \pm 0.15$ & $35.56 \pm 1.54$ & $36.34 \pm 1.39$ & 0.40 (79\%) & $0.41(80 \%)$ & 0.81 & 0.83 & 1.33 & 1.35 \\
\hline 92 & $\mathrm{~Pa} 3$ & $3.80 \pm 0.17$ & $3.63 \pm 0.16$ & $39.09 \pm 1.42$ & $37.60 \pm 1.00$ & $0.45(88 \%)$ & $0.43(85 \%)$ & 0.86 & 0.85 & 1.31 & 1.29 \\
\hline
\end{tabular}

The same glucose concentrations were supplemented to YNB as reference broth. The highest glycerol and ethanol levels were reported. All experiments were conducted in triplicate $( \pm S D)$. Y $Y_{E}$, ethanol yield per gram of consumed glucose calculated on the highest ethanol production and \% of theoretical maximum indicated in brackets; $Q$, volumetric productivity at the highest ethanol production.

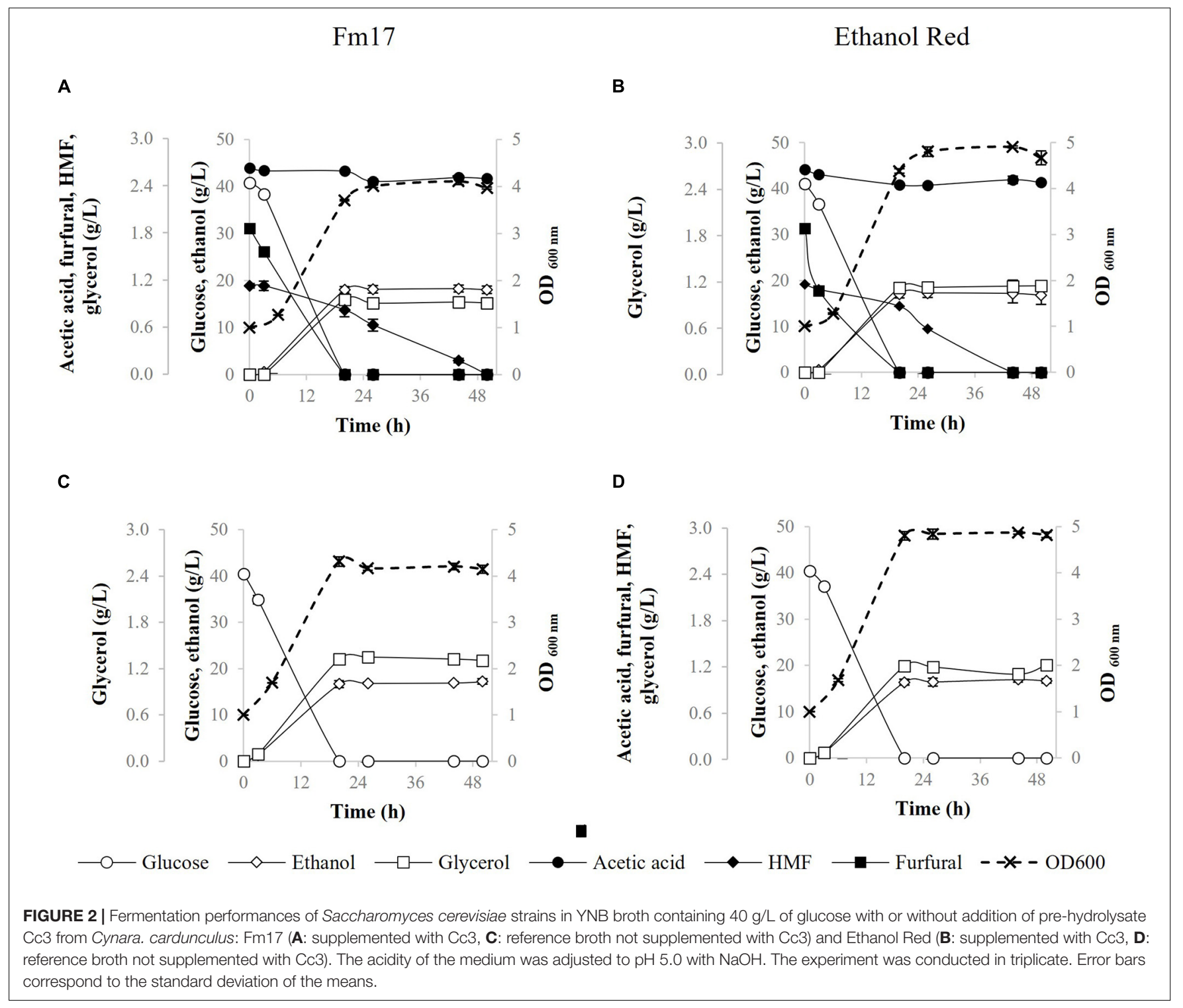


Fm17

A

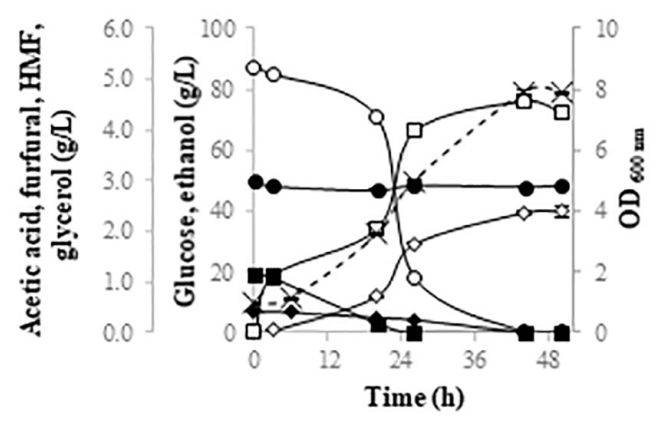

C

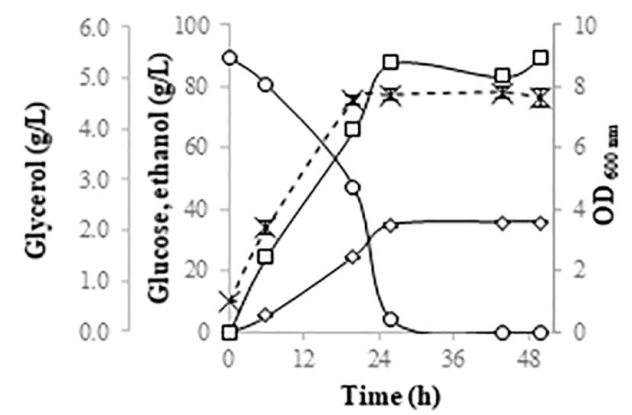

Ethanol Red

B

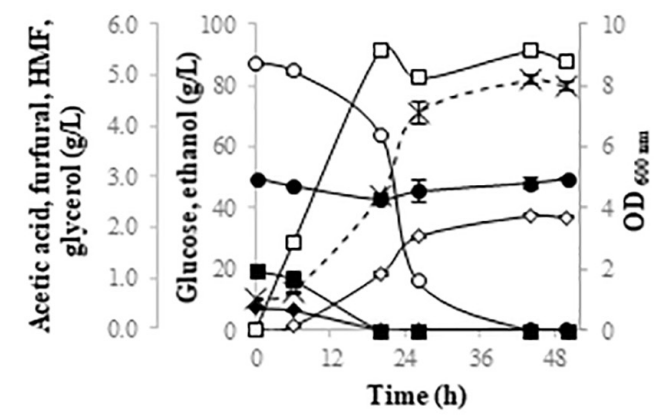

D

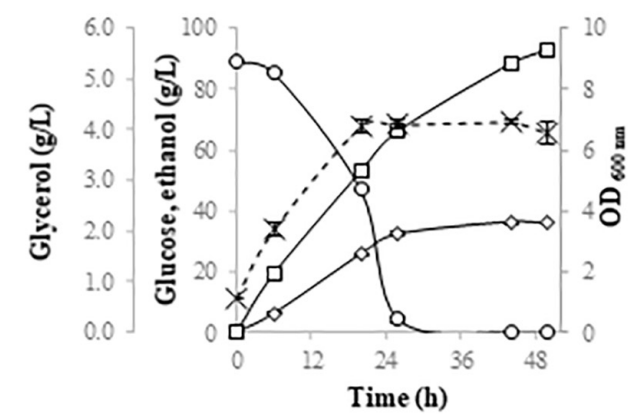

me (h)

$\multimap$ Glucose $\neg$ Ethanol $\rightarrow-$ Glycerol

$\longrightarrow$ Acetic acid

$\longrightarrow$ HMF $\rightarrow-$ Furfural $\quad-\not--$ OD600

FIGURE 3 | Fermentation performances of Saccharomyces cerevisiae strains in YNB broth containing $92 \mathrm{~g} / \mathrm{L}$ of glucose with or without the addition of pre-hydrolysate Pa3 from Phragmites australis: Fm17 (A: supplemented with Pa3, C: reference broth not supplemented with Pa3) and Ethanol Red (B: supplemented with $\mathrm{Cc3}$, D: reference broth not supplemented with $\mathrm{Pa3}$ ). The acidity of the medium was adjusted to $\mathrm{pH} 5.0 \mathrm{with} \mathrm{NaOH}$. The experiment was conducted in triplicate. Error bars correspond to the standard deviation of the means.

While all S. cerevisiae isolates showed similar tolerance to Cc4, amounting to about $60 \%$ relative growth (54-66\%), strong differences could be identified in the case of the liquor $\mathrm{Pa} 3$ (8-69\%) and Cc3 (57-88\%).

The reference industrial yeast Ethanol Red proved to be extremely inhibited by $\mathrm{Pa} 3$ while showing high tolerance in Cc3 and Cc4. On the contrary, S. cerevisiae Fm17 exhibited the highest relative growth values once exposed to the three pre-hydrolysates at $\mathrm{pH}$ 5.0. Similar inhibitor tolerance patterns, although with lower values, were detected for S. cerevisiae Fm90 and YI30 (Table 5).

Benefits generated by $\mathrm{pH}$ adjustment can be ascribed to the acidity-related dissociation of weak acids. As extracellular undissociated acids are liposoluble, they can permeate through the cell membrane and lower the cytosolic $\mathrm{pH}$, thus inducing stress levels to the cell that can cause the inhibition of metabolic activities. The amount of dissociated acid is a function of $\mathrm{pH}$ and the $\mathrm{p} K_{a}$ of each specific acid. The concentration of undissociated and dissociated acids in lignocellulosic pre-hydrolysates is then very sensitive to the medium acidity (Palmqvist and HahnHägerdal, 2000; Landaeta et al., 2013; Jönsson and Martín, 2016). The increase of medium $\mathrm{pH}$ to values closer to, or higher than, the $\mathrm{p} K_{a}$ of weak acids reduces the concentration of harmful undissociated acids, resulting in less stressful conditions for the yeast. This is particularly true for the hydrolysate Cc4, quite rich in terms of formic and acetic acid (Table 1).

Based on the high inhibitor tolerance shown in different lignocellulosic pre-hydrolysates, S. cerevisiae Fm17 was selected and further characterized, together with the reference Ethanol Red, in terms of fermenting abilities in the lignocellulosic pretreatment liquors of $P$. australis $(\mathrm{Pa} 3)$ and $C$. cardunculus (Cc3).

\section{Fermentation Performances of Selected Yeast Strains in Lignocellulosic Pre-hydrolysates}

Since developing industrial yeast with high fermentative capacity from different pre-treated feedstocks, rather than a preferred substrate, is one of the ultimate goals, pre-hydrolysates from cardoon $(\mathrm{Cc} 3)$ and common reed $(\mathrm{Pa} 3)$ were used as a substrate to simulate the industrial environment as closely as possible. Firstly, the pre-treatment liquors were supplemented up to $20 \mathrm{~g} / \mathrm{L}$ glucose and used in small-scale fermentations to compare the fermenting abilities of S. cerevisiae Fm17 to those of the reference 
industrial strain Ethanol Red (Figures 1A-D and Table 6). The acidity of the medium was adjusted to $\mathrm{pH} 5.0$ with $5 \mathrm{M} \mathrm{NaOH}$. A fermentation medium formulated without $\mathrm{Cc} 3$ or $\mathrm{Pa} 3$ was used as control (Figures 1E,F and Table 6).

Once exposed to the pre-hydrolysate Cc3 (C. cardunculus), glucose consumption was completed within the first $20 \mathrm{~h}$ by both strains (Figures 1A,B). The industrial yeast Ethanol Red produced higher biomass levels with the final $\mathrm{OD}_{600}$ approaching 4.0. On the contrary, the novel yeast strain Fm17 achieved higher ethanol production $(9.34 \mathrm{~g} / \mathrm{L})$ which was 1.13 -fold that of Ethanol Red (Figures 1A,B and Table 6). As such, ethanol yield and maximum productivity $\left(Q_{\max }\right)$ values were greater (Table 6). In particular, the selected S. cerevisiae Fm17 exhibited an ethanol yield of $0.46 \mathrm{~g} / \mathrm{g}$ of consumed glucose, corresponding to almost $90 \%$ of the theoretical $(0.51 \mathrm{~g} / \mathrm{g})$, whereas the industrial benchmark stopped only at $0.40 \mathrm{~g} / \mathrm{g}$, which corresponds to $79 \%$ of the maximum yield.

The strains produced similar fermenting patterns also in the presence of the pre-treatment liquor $\mathrm{Pa} 3$ from $P$. australis (Figures 1C,D and Table 6). Biomass yield, detected as $\mathrm{OD}_{600^{-}}$ values, was higher in the case of $S$. cerevisiae Ethanol Red (Figures 1C,D); meanwhile, ethanol performances were better for the selected strain Fm17 (Figures 1C,D and Table 6), which produced up to 9.04 instead of $8.01 \mathrm{~g} / \mathrm{L}$. The resulting ethanol yields were 0.45 and $0.40 \mathrm{~g} / \mathrm{g}$ of consumed glucose, corresponding to 88 and $78 \%$ of the theoretical for S. cerevisiae Fm17 and Ethanol Red, respectively (Table 6).

In the control medium, YNB with $20 \mathrm{~g} / \mathrm{L}$ of glucose (Figures 1E,F), S. cerevisiae Fm17, and Ethanol Red readily consumed all the glucose available and $\mathrm{OD}_{600}$ levels were higher than those detected in the presence of both $\mathrm{Cc} 3$ and $\mathrm{Pa}$. Furthermore, Ethanol Red reached $\mathrm{OD}_{600}$ levels greater than those of Fm17 with values of almost 5.0 (Figures 1E,F). Noteworthy, ethanol levels produced by both strains were lower than those detected in the pre-hydrolysates. S. cerevisiae Fm17 and Ethanol Red yielded 8.17 and $8.21 \mathrm{~g} / \mathrm{L}$ of ethanol, corresponding to 80 and $81 \%$ of the theoretical, respectively (Figures 1C,D). Since pre-hydrolysates have a complex chemical composition, presence of additional carbon sources in the medium containing $\mathrm{Cc} 3$ and $\mathrm{Pa} 3$ is possible, resulting in greater ethanol productions. Furthermore, a higher amount of ethanol produced in the pre-hydrolysates can also be ascribed to the presence of furfural and HMF. Although these chemical compounds exhibit a negative impact on yeast metabolism, their reduction to less toxic compounds can act as a redox sink, thus preventing redox imbalances and increasing final ethanol yield (Wahlbom and HahnHägerdal, 2002; Ask et al., 2013; Favaro et al., 2013a). Furfural and HMF were completely metabolized by the strains (Figures 1A-D). Lower glycerol production observed in Cc3 and $\mathrm{Pa} 3$ when compared to the control broth further supports this hypothesis, since glycerol production as redox sink is less favored than furan conversion (Palmqvist et al., 1999; Martín and Jönsson, 2003).

As reported in Table 6, in the presence of both pre-treatment liquors, the volumetric productivities of the yeast strains were generally greater than those recorded in the reference medium (without inhibitor supplementation). This could be due to the presence of weak acids, which can boost the fermentation rate at concentrations below 100 mM (Palmqvist and Hahn-Hägerdal, 2000; Favaro et al., 2013a; Jönsson et al., 2013; Jönsson and Martín, 2016).

To further assess the fermenting abilities of both strains in industrially relevant conditions, their performances were evaluated in YNB medium formulated with Cc3 (Figure 2) or Pa3 (Figure 3) pre-hydrolysates supplemented up to 40 and $92 \mathrm{~g} / \mathrm{L}$ of glucose, respectively, to mimic the highest glucose concentration obtained by enzymatic saccharification of each steam-exploded WIS (Cotana et al., 2015a,b; Cavalaglio et al., 2016). pHvalue was adjusted to 5.0 with $5 \mathrm{M} \mathrm{NaOH}$. Fermentation medium formulated without $\mathrm{Cc} 3$ or $\mathrm{Pa} 3$ was used as control (Figures 2C,D, 3C,D).

In the presence of the pre-hydrolysate Cc3 (C. cardunculus) and $40 \mathrm{~g} / \mathrm{L}$ glucose, the strains utilized all glucose available by $20 \mathrm{~h}$ of fermentation (Figures 2A,B). Ethanol Red produced higher biomass than Fm17 in both broths (with and without $\mathrm{Cc} 3$ ): final $\mathrm{OD}_{600}$ was 4.9 in the control medium and 4.8 in presence of $\mathrm{Cc} 3$, amounting to $16 \%$ and $20 \%$ higher than $\mathrm{Fm} 17$, respectively (Figure 2). On the contrary, Fm17 displayed better volumetric productivities and ethanol production (Figure 2 and Table 6). Fm17 and Ethanol Red produced $18.29 \mathrm{~g} / \mathrm{L}$ and $17.25 \mathrm{~g} / \mathrm{L}$ of ethanol in the medium formulated with Cc3, respectively, corresponding to $88 \%$ and $82 \%$ of the theoretical yield (Figures 2A,B and Table 6). Volumetric productivities of Fm17 was significantly higher than those of the industrial yeast, with $Q_{\max }$ values of 0.90 instead of $0.85\left(\mathrm{~g} / \mathrm{L} \mathrm{h}^{-1}\right)$ for $S$. cerevisiae Fm17 and Ethanol Red, respectively (Table 6).

In terms of furans reduction, furfural and HMF were completely metabolized by both strains (Figure 2). In the control medium supplemented with $40 \mathrm{~g} / \mathrm{L}$ glucose, S. cerevisiae Fm17 and Ethanol Red produced lower ethanol levels: 16.92 and $16.95 \mathrm{~g} / \mathrm{L}$ of ethanol, respectively, corresponding to $82 \%$ of the theoretical (Figures 2C,D and Table 6). This finding is consistent with the ethanol performances described earlier (Figure 1), further supporting the hypothesis that the occurrence of additional carbon sources and/or redox sinks in the pre-hydrolysate may have enhanced ethanol production by both strains.

Once exposed to the pre-hydrolysate $\mathrm{Pa} 3$ ( $P$. australis) with $92 \mathrm{~g} / \mathrm{L}$ glucose, the strains confirmed their ability to withstand high inhibitor concentrations (Figures 3A,B). However, glucose consumption of both strains took longer than in the reference medium (Figures 3C,D). Nevertheless, ethanol production was high with $S$. cerevisiae Fm17 having again the most promise $(39.09 \mathrm{~g} / \mathrm{L})$ corresponding to about $88 \%$ of the theoretical. The volumetric productivity values of both strains were comparable (Table 6).

As already described and discussed above, ethanol levels in $\mathrm{Pa} 3$ were again higher than those detected in the reference broth (YNB supplemented with $92 \mathrm{~g} / \mathrm{L}$ ). S. cerevisiae Fm17 and Ethanol Red quickly converted glucose to comparable amounts of alcohol ( 35.56 and $36.34 \mathrm{~g} / \mathrm{L}$, respectively), with an ethanol yield of about $80 \%$ of the theoretical (Figures 3C,D and Table 6). 
Taken together, the results of small-scale fermentations in the presence of two pre-hydrolysates and increasing concentrations of glucose showed that $S$. cerevisiae Fm17 outcompeted ethanol performances of the reference strain Ethanol Red currently used in industrial bioethanol production (Figures 1-3 and Table 6). Interestingly, the lower glucose concentrations, the greater ethanol yields and productivities $(p \leq 0.05)$ were achieved by the superior yeast $S$. cerevisiae Fm17. Glycerol levels were comparable in both strain fermentations; meanwhile, biomass yields were always higher in the case of the industrial benchmark yeast (Figures 1-3). These findings could be explained considering the ecological origin of Fm17, which has been isolated from grape marcs, an extreme environment with a limited amount of glucose (Favaro et al., 2013a). On the contrary, the industrial strain Ethanol Red has been specifically selected for high alcohol yield and tolerance especially during very high gravity fermentation, typical of the corn ethanol industry where at least $200 \mathrm{~g} / \mathrm{L}$ glucose is available (Walker and Walker, 2018). As such, the novel S. cerevisiae Fm17 seems to be able to withstand better the inhibitors at lower glucose concentrations, reducing biomass yield in favor of ethanol production. These observations are in agreement with the fact that, under anaerobic conditions, yeast cells use alcoholic fermentation of sugars as the sole pathway to obtain energy in the form of ATP for cellular maintenance and, if sufficient ATP is available, for growth. When ATP is utilized for growth, yeast biomass and associated glycerol are produced at the expense of sugars that are not converted to alcohol (Gombert and van Maris, 2015). Furthermore, considering that under SSF or Consolidated BioProcessing settings, glucose levels, released by commercial or, respectively, recombinant enzymes produced by the engineered yeast, do not usually accumulate because of the quick yeast utilization (Hasunuma and Kondo, 2012; Cripwell et al., 2019, 2020), Fm17 should be considered as a very promising lignocellulosic ethanol strain.

In conclusion, this paper was successful in employing undetoxified steam-exploded lignocellulosic residues for secondgeneration ethanol production. The liquors were used in a close simulation of industrial conditions, considering that as the key point for strain selection.

A cluster of yeast strains demonstrated inhibitor tolerance higher than those of $S$. cerevisiae Ethanol Red, the most used microorganism for lignocellulosic ethanol. This finding is of great value considering that to obtain large additional profits, firstgeneration ethanol plants strive for an increase of even $1 \%$ in ethanol yield. Techno-economical evaluations are in progress to determine the weight of using S. cerevisiae Fm17 in the overall process efficiency. Moreover, further studies are on-going to confirm its promising industrial fitness both at higher scale (i.e., bioreactor) and in SSF settings in the presence of WIS collected after steam-explosion of selected lignocellulosic materials.

This study also implies that there are interesting opportunities to isolate or engineer natural yeast variants with performances better than those currently exploited in well-known industrial yeast strains. Moreover, the phenotypic differences between the screened yeast in terms of inhibitor-tolerance indicated that the choice of strain is critical when contemplating the design of a process involving fermentation of lignocellulosic pre-treated materials at industrial scale.

The most burgeoning strain, capable of growing well in undiluted liquors, was also able to ferment in more than one pretreated feedstocks. This confirmed its great versatility to multiple pre-treated materials, which is one of the requirements for an efficient second-generation ethanol yeast.

\section{DATA AVAILABILITY STATEMENT}

The original contributions presented in the study are included in the article/Supplementary Material, further inquiries can be directed to the corresponding author/s.

\section{AUTHOR CONTRIBUTIONS}

LC: investigation, data curation, writing original draft, and visualization. NG: data curation, writing original draft, and visualization. $\mathrm{MB}$ and SC: commenting revised draft and funding acquisition. LF: conceptualization, methodology, data curation, reviewing original draft, editing, visualization, supervision, and funding acquisition. All authors contributed to the article and approved the submitted version.

\section{FUNDING}

This work was supported by University of Padova [grants GRIC120EG8, DOR1715524/17, DOR1728499/17, DOR1824847/18, DOR1827441/18, DOR1931153/19, DOR1928058/19, DOR2087054/20, DOR2084579/20, DOR2027838/20, and BIRD210708/21].

\section{ACKNOWLEDGMENTS}

The authors are grateful to Valentino Pizzocchero, M.Sc. (University of Padova, Italy) for HPLC analysis and Elisabetta Bordignon, M.Sc. (University of Padova) for valuable analytical support. Prof. Willem H. van Zyl and Dr. Trudy Jansen (University of Stellenbosch, South Africa) are gratefully acknowledged for providing $S$. cerevisiae $\mathrm{M} 2 \mathrm{n}$ and YI30. Sugarcane pre-hydrolysate has been kindly provided by Prof. Johann F. Görgens (University of Stellenbosch). Dr. Gianluca Cavalaglio, Prof. Andrea Nicolini, and Prof. Franco Cotana (University of Perugia, Italy) are also gratefully acknowledged for providing common reed and cardoon pre-hydrolysates.

\section{SUPPLEMENTARY MATERIAL}

The Supplementary Material for this article can be found online at: https://www.frontiersin.org/articles/10.3389/fmicb.2021. 756032/full\#supplementary-material 


\section{REFERENCES}

Abdel-Banat, B. M. A., Hoshida, H., Ano, A., Nonklang, S., and Akada, R. (2010). High-temperature fermentation: How can processes for ethanol production at high temperatures become superior to the traditional process using mesophilic yeast? Appl. Microbiol. Biotechnol. 85, 861-867. doi: 10.1007/s00253-009-2 248-5

Albers, E., and Larsson, C. (2009). A comparison of stress tolerance in YPD and industrial lignocellulose-based medium among industrial and laboratory yeast strains. J. Ind. Microbiol. Biotechnol. 36, 1085-1091. doi: 10.1007/s10295-0090592-1

Ask, M., Bettiga, M., Mapelli, V., and Olsson, L. (2013). The influence of HMF and furfural on redox-balance and energy-state of xylose-utilizing Saccharomyces cerevisiae. Biotechnol. Biofuels 6, 1-13. doi: 10.1186/1754-6834-6-22

Awasthi, M. K., Sarsaiya, S., Patel, A., Juneja, A., Singh, R. P., Yan, B., et al. (2020). Refining biomass residues for sustainable energy and bio-products: an assessment of technology, its importance, and strategic applications in circular bio-economy. Renew. Sustain. Energy Rev. 127:109876. doi: 10.1016/j.rser.2020. 109876

Basso, L. C., De Amorim, H. V., De Oliveira, A. J., and Lopes, M. L. (2008). Yeast selection for fuel ethanol production in Brazil. FEMS Yeast Res. 8, 1155-1163. doi: 10.1111/j.1567-1364.2008.00428.x

Bhatia, S. K., Kim, S. H., Yoon, J. J., and Yang, Y. H. (2017). Current status and strategies for second generation biofuel production using microbial systems. Energy Convers. Manage. 148, 1142-1156. doi: 10.1016/j.enconman.2017.06. 073

Bhutto, A. W., Qureshi, K., Harijan, K., Abro, R., Abbas, T., Bazmi, A. A., et al. (2017). Insight into progress in pre-treatment of lignocellulosic biomass. Energy 122, 724-745. doi: 10.1016/j.energy.2017.01.005

Brandt, B. A., Jansen, T., Görgens, J. F., and van Zyl, W. H. (2019). Overcoming lignocellulose-derived microbial inhibitors: advancing the Saccharomyces cerevisiae resistance toolbox. Biofuels Bioprod. Biorefining 13, 1520-1536. doi: 10.1002/bbb. 2042

Bułkowska, K., and Klimiuk, E. (2016). "Pretreatment of lignocellulosic biomass," in Biomass for Biofuels, eds K. Bulkowska, Z. Mariusz Gusiatin, E. Klimiuk, A. Pawlowski, and T. Pokoj (London: CRC Press), 129-162. doi: 10.1201/ 9781315226422

Cagnin, L., Favaro, L., Pizzocchero, V., Cotana, F., Nicolini, A., Cavalaglio, G., et al. (2018). "Novel yeast strains for the efficient conversion of steam-exploded lignocellulosic waste streams to bioethanol," in Proceedings of the NAXOS 2018 6th International Conference on Sustainable Solid Waste Management, Naxos.

Cavalaglio, G., Gelosia, M., Ingles, D., Pompili, E., D’Antonio, S., and Cotana, F. (2016). Response surface methodology for the optimization of cellulosic ethanol production from Phragmites australis through pre-saccharification and simultaneous saccharification and fermentation. Ind. Crop. Prod. 83, 431-437. doi: 10.1016/j.indcrop.2015.12.089

Chandel, A. K., Garlapati, V. K., Singh, A. K., Antunes, F. A. F., and da Silva, S. S. (2018). The path forward for lignocellulose biorefineries: bottlenecks, solutions, and perspective on commercialization. Bioresour. Technol. 264, 370-381. doi: 10.1016/j.biortech.2018.06.004

Cotana, F., Cavalaglio, G., Gelosia, M., Coccia, V., Petrozzi, A., Ingles, D., et al. (2015a). A comparison between SHF and SSSF processes from cardoon for ethanol production. Ind. Crop. Prod. 69, 424-432. doi: 10.1016/j.indcrop.2015. 02.064

Cotana, F., Cavalaglio, G., Pisello, A., Gelosia, M., Ingles, D., and Pompili, E. (2015b). Sustainable Ethanol Production from Common Reed (Phragmites australis) through Simultaneuos Saccharification and Fermentation. Sustainability 7, 12149-12163. doi: 10.3390/su70912149

Cripwell, R. A., Favaro, L., Viljoen-Bloom, M., and van Zyl, W. H. (2020). Consolidated bioprocessing of raw starch to ethanol by Saccharomyces cerevisiae: achievements and challenges. Biotechnol. Adv. 42:107579. doi: 10 . 1016/j.biotechadv.2020.107579

Cripwell, R. A., Rose, S. H., Favaro, L., and Van Zyl, W. H. (2019). Construction of industrial Saccharomyces cerevisiae strains for the efficient consolidated bioprocessing of raw starch. Biotechnol. Biofuels 12, 1-16. doi: 10.1186/s13068019-1541-5

Cunha, J. T., Romaní, A., Costa, C. E., Sá-Correia, I., and Domingues, L. (2019). Molecular and physiological basis of Saccharomyces cerevisiae tolerance to adverse lignocellulose-based process conditions. Appl. Microbiol. Biotechnol. 103, 159-175. doi: 10.1007/s00253-018-9478-3

da Costa Sousa, L., Chundawat, S. P. S., Balan, V., and Dale, B. E. (2009). 'Cradleto-grave' assessment of existing lignocellulose pretreatment technologies. Curr. Opin. Biotechnol. 20, 339-347. doi: 10.1016/j.copbio.2009.05.003

Dale, B. (2018). Time to rethink cellulosic biofuels? Biofuel. Bioprod. Biorefin. 12, 5-7. doi: 10.1002/bbb.1856

de Witt, R. N., Kroukamp, H., and Volschenk, H. (2019). Proteome response of two natural strains of Saccharomyces cerevisiae with divergent lignocellulosic inhibitor stress tolerance. FEMS Yeast Res. 19, foy116. doi: 10.1093/femsyr/ foyl16

Della-Bianca, B. E., Basso, T. O., Stambuk, B. U., Basso, L. C., and Gombert, A. K. (2013). What do we know about the yeast strains from the Brazilian fuel ethanol industry? Appl. Microbiol. Biotechnol. 97, 979-991. doi: 10.1007/s00253-0124631-x

Dmytruk, K. V., Kurylenko, O. O., Ruchala, J., Abbas, C. A., and Sibirny, A. A. (2017). "Genetic improvement of conventional and nonconventional yeasts for the production of first- and second-generation ethanol," in Biotechnology of Yeasts and Filamentous Fungi, ed. A. Sibirny (Cham: Springer). doi: 10.1007/ 978-3-319-58829-2_1

Dubey, R., Jakeer, S., and Gaur, N. A. (2016). Screening of natural yeast isolates under the effects of stresses associated with second-generation biofuel production. J. Biosci. Bioeng. 121, 509-516. doi: 10.1016/j.jbiosc.2015.09.006

Espada, J. J., Villalobos, H., and Rodríguez, R. (2021). Environmental assessment of different technologies for bioethanol production from Cynara cardunculus: a life cycle assessment study. Biomass Bioenergy 144: 105910. doi: 10.1016/j. biombioe.2020.105910

Espirito Santo, M. C., Fockink, D. H., Pellegrini, V. O. A., Guimaraes, F. E. G., deAzevedo, E. R., Ramos, L. P., et al. (2020). Physical techniques shed light on the differences in sugarcane bagasse structure subjected to steam explosion pretreatments at equivalent combined severity factors. Ind. Crop. Prod. 158:113003. doi: 10.1016/j.indcrop.2020.113003

Favaro, L., Basaglia, M., and Casella, S. (2014). Innately robust yeast strains isolated from grape marc have a great potential for lignocellulosic ethanol production. Ann. Microbiol. 64, 1807-1818. doi: 10.1007/s13213-014-0826-y

Favaro, L., Basaglia, M., Saayman, M., Rose, S. H., van Zyl, W. H., and Casella, S. (2010). Engineering amylolytic yeasts for industrial bioethanol production. Chem. Eng. Trans. 20, 97-102. doi: 10.3303/CET1020017

Favaro, L., Basaglia, M., van Zyl, W. H., and Casella, S. (2013b). Using an efficient fermenting yeast enhances ethanol production from unfiltered wheat bran hydrolysates. Appl. Energy 102, 170-178. doi: 10.1016/j.apenergy.2012.05.059

Favaro, L., Basaglia, M., Trento, A., Van Rensburg, E., García-Aparicio, M., Van Zyl, W. H., et al. (2013a). Exploring grape marc as trove for new thermotolerant and inhibitor-tolerant Saccharomyces cerevisiae strains for second-generation bioethanol production. Biotechnol. Biofuels 6, 1-14. doi: 10.1186/1754-6834-6168

Favaro, L., Jansen, T., and van Zyl, W. H. (2019b). Exploring industrial and natural Saccharomyces cerevisiae strains for the bio-based economy from biomass: the case of bioethanol. Crit. Rev. Biotechnol. 39, 800-816. doi: 10.1080/07388551. 2019.1619157

Favaro, L., Cagnin, L., Corte, L., Roscini, L., De Pascale, F., Treu, L., et al. (2019a). Metabolomic alterations do not induce metabolic burden in the industrial yeast M2n [pBKD2-Pccbgl1]-C1 engineered by multiple $\delta$-integration of a fungal $\beta$-glucosidase gene. Front. Bioeng. Biotechnol. 7:376. doi: 10.3389/fbioe.2019. 00376

Favaro, L., Corte, L., Roscini, L., Cagnin, L., Tiecco, M., Colabella, C., et al. (2016). A novel FTIR-based approach to evaluate the interactions between lignocellulosic inhibitory compounds and their effect on yeast metabolism. RSC Adv. 6, 47981-47989. doi: 10.1039/c6ra $08859 \mathrm{~g}$

Fockink, D. H., Sánchez, J. H., and Ramos, L. P. (2018). Comprehensive analysis of sugarcane bagasse steam explosion using autocatalysis and dilute acid hydrolysis (H3PO4 and $\mathrm{H} 2 \mathrm{SO} 4)$ at equivalent combined severity factors. Ind. Crop. Prod. 123, 563-572. doi: 10.1016/j.indcrop.2018.07.017

Galbe, M., and Zacchi, G. (2007). "Pretreatment of lignocellulosic materials for efficient bioethanol production," in Biofuels. Advances in Biochemical Engineering/Biotechnology, Vol. 108, ed. L. Olsson (Berlin: Springer), 41-65. doi: 10.1007/10_2007_070 
Garay-Arroyo, A., Covarrubias, A. A., Clark, I., Niño, I., Gosset, G., and Martinez, A. (2004). Response to different environmental stress conditions of industrial and laboratory Saccharomyces cerevisiae strains. Appl. Microbiol. Biotechnol. 63, 734-741. doi: 10.1007/s00253-003-1414-4

García, A., González Alriols, M., and Labidi, J. (2014). Evaluation of different lignocellulosic raw materials as potential alternative feedstocks in biorefinery processes. Ind. Crop. Prod. 53, 102-110. doi: 10.1016/j.indcrop.2013.12.019

Gombert, A. K., and van Maris, A. J. A. (2015). Improving conversion yield of fermentable sugars into fuel ethanol in 1st generation yeast-based production processes. Curr. Opin. Biotechnol. 33, 81-86. doi: 10.1016/j.copbio.2014.12.012

Hasunuma, T., and Kondo, A. (2012). Consolidated bioprocessing and simultaneous saccharification and fermentation of lignocellulose to ethanol with thermotolerant yeast strains. Process Biochem. 47, 1287-1294. doi: 10.1016/ j.procbio.2012.05.004

Huang, S., Liu, T., Peng, B., and Geng, A. (2019). Enhanced ethanol production from industrial lignocellulose hydrolysates by a hydrolysate-cofermenting Saccharomyces cerevisiae strain. Bioprocess Biosyst. Eng. 42, 883-896. doi: 10. 1007/s00449-019-02090-0

Jansen, M. L. A., Bracher, J. M., Papapetridis, I., Verhoeven, M. D., de Bruijn, H., de Waal, P. P., et al. (2017). Saccharomyces cerevisiae strains for second-generation ethanol production: from academic exploration to industrial implementation. FEMS Yeast Res. 17, 1-20. doi: 10.1093/femsyr/fox044

Jansen, T., Hoff, J. W., Jolly, N., and van Zyl, W. H. (2018). Mating of natural Saccharomyces cerevisiae strains for improved glucose fermentation and lignocellulosic inhibitor tolerance. Folia Microbiol. 63, 155-168. doi: 10.1007/ s12223-017-0546-3

Jönsson, L. J., Alriksson, B., and Nilvebrant, N. O. (2013). Bioconversion of lignocellulose: inhibitors and detoxification. Biotechnol. Biofuels 6:16. doi: 10 . 1186/1754-6834-6-16

Jönsson, L. J., and Martín, C. (2016). Pretreatment of lignocellulose: formation of inhibitory by-products and strategies for minimizing their effects. Bioresour. Technol. 199, 103-112. doi: 10.1016/j.biortech.2015.10.009

Kádár, Z., Maltha, S. F., Szengyel, Z., Réczey, K., and De Laat, W. (2007). Ethanol fermentation of various pretreated and hydrolyzed substrates at low initial $\mathrm{pH}$. Appl. Biochem. Biotechnol. 137-140, 847-858. doi: 10.1007/s12010-007-9102-y

Kim, S. R., Skerker, J. M., Kong, I. I., Kim, H., Maurer, M. J., Zhang, G.-C., et al. (2017). Metabolic engineering of a haploid strain derived from a triploid industrial yeast for producing cellulosic ethanol. Metab. Eng. 40, 176-185. doi: 10.1016/j.ymben.2017.02.006

Landaeta, R., Aroca, G., Acevedo, F., Teixeira, J. A., and Mussatto, S. I. (2013). Adaptation of a flocculent Saccharomyces cerevisiae strain to lignocellulosic inhibitors by cell recycle batch fermentation. Appl. Energy 102, 124-130. doi: 10.1016/j.apenergy.2012.06.048

Liu, C. G., Xiao, Y., Xia, X. X., Zhao, X. Q., Peng, L., Srinophakun, P., et al. (2019). Cellulosic ethanol production: progress, challenges and strategies for solutions. Biotechnol. Adv. 37, 491-504. doi: 10.1016/j.biotechadv.2019.03.002

Liu, Y. J., Li, B., Feng, Y., and Cui, Q. (2020). Consolidated bio-saccharification: leading lignocellulose bioconversion into the real world. Biotechnol. Adv. 40:107535. doi: 10.1016/j.biotechadv.2020.107535

Martín, C., and Jönsson, L. J. (2003). Comparison of the resistance of industrial and laboratory strains of Saccharomyces and Zygosaccharomyces to lignocellulosederived fermentation inhibitors. Enzyme Microb. Technol. 32, 386-395. doi: 10.1016/S0141-0229(02)00310-1

Martìn, C., Galbe, M., Wahlbom, C. F., Hahn-Hägerdal, B., and Jönsson, L. J. (2002). Ethanol production from enzymatic hydrolysates of sugarcane bagasse using recombinant xylose-utilising Saccharomyces cerevisiae. Enzyme Microb. Technol. 31, 274-282. doi: 10.1016/S0141-0229(02)00112-6

Mertens, J. A., Kelly, A., and Hector, R. E. (2018). Screening for inhibitor tolerant Saccharomyces cerevisiae strains from diverse environments for use as platform strains for production of fuels and chemicals from biomass. Bioresour. Technol. Rep. 3, 154-161. doi: 10.1016/j.biteb.2018.07.006

Morales, P., Gentina, J. C., Aroca, G., and Mussatto, S. I. (2017). Development of an acetic acid tolerant Spathaspora passalidarum strain through evolutionary engineering with resistance to inhibitors compounds of autohydrolysate of Eucalyptus globulus. Ind. Crop. Prod. 106, 5-11. doi: 10.1016/j.indcrop.2016. 12.023

Mussatto, S. I. (2016). Biomass Fractionation Technologies for a Lignocellulosic Feedstock Based Biorefinery. Amsterdam: Elsevier. doi: 10.1016/C2014-001890-4
Nair, R. B., Lennartsson, P. R., and Taherzadeh, M. J. (2017). "Bioethanol production from agricultural and municipal wastes," in Current Developments in Biotechnology and Bioengineering, eds J. Wong, R. D. Tyagi and A. Pandey (Amsterdam: Elsevier), 157-190.

Overend, R. P., and Chornet, E. (1987). Fractionation of lignocellulosics by steamaqueous pretreatments. Philos. Trans. R. Soc. Lond. Ser. A 321, 523-536. doi: 10.1098/rsta.1987.0029

Palmqvist, E., Almeida, J. S., and Hahn-Hägerdal, B. (1999). Influence of furfural on anaerobic glycolytic kinetics of Saccharomyces cerevisiae in batch culture. Biotechnol. Bioeng. 62, 447-454.

Palmqvist, E., and Hahn-Hägerdal, B. (2000). Fermentation of lignocellulosic hydrolysates. I: Inhibition and detoxification. Bioresour. Technol. 74, 17-24. doi: 10.1016/S0960-8524(99)00160-1

Park, H., Jeong, D., Shin, M., Kwak, S., Oh, E. J., Ko, J. K., et al. (2020). Xylose utilization in Saccharomyces cerevisiae during conversion of hydrothermally pretreated lignocellulosic biomass to ethanol. Appl. Microbiol. Biotechnol. 104, 3245-3252. doi: 10.1007/s00253-020-10 427-z

Pereira, F. B., Guimarães, P. M. R., Teixeira, J. A., and Domingues, L. (2011). Robust industrial Saccharomyces cerevisiae strains for very high gravity bioethanol fermentations. J. Biosci. Bioeng. 112, 130-136. doi: 10.1016/j.jbiosc. 2011.03.022

Pereira, F. B., Romaní, A., Ruiz, H. A., Teixeira, J. A., and Domingues, L. (2014). Industrial robust yeast isolates with great potential for fermentation of lignocellulosic biomass. Bioresour. Technol. 161, 192-199. doi: 10.1016/j. biortech.2014.03.043

Roscini, L., Favaro, L., Corte, L., Cagnin, L., Colabella, C., Basaglia, M., et al. (2019). A yeast metabolome-based model for an ecotoxicological approach in the management of lignocellulosic ethanol stillage. R. Soc. Open Sci. 6:180718. doi: $10.1098 /$ rsos. 180718

van Dijk, M., Trollmann, I., Fontes Saraiva, M. A., Lopes Brandão, R., Olsson, L., and Nygård, Y. (2020). Small scale screening of yeast strains enables high-throughput evaluation of performance in lignocellulose hydrolysates. Bioresour. Technol. Rep. 11:100532. doi: 10.1016/j.biteb.2020.10 0532

Viktor, M. J., Rose, S. H., Van Zyl, W. H., and Viljoen-Bloom, M. (2013). Raw starch conversion by Saccharomyces cerevisiae expressing Aspergillus tubingensis amylases. Biotechnol. Biofuels 6:167. doi: 10.1186/1754-6834-6-167

Wahlbom, C. F., and Hahn-Hägerdal, B. (2002). Furfural, 5-hydroxymethyl furfural, and acetoin act as external electron acceptors during anaerobic fermentation of xylose in recombinant Saccharomyces cerevisiae. Biotechnol. Bioeng. 78, 172-178. doi: 10.1002/bit.10188

Walker, G. M., and Walker, R. S. K. (2018). "Enhancing yeast alcoholic fermentations," in Advances in Applied Microbiology, Vol. 105, eds G. M. Gadd and S. Sariaslani (Cambridge, MA: Academic Press), 87-129. doi: 10.1016/bs. aambs.2018.05.003

Wimalasena, T. T., Greetham, D., Marvin, M. E., Liti, G., Chandelia, Y., Hart, A., et al. (2014). Phenotypic characterisation of Saccharomyces spp. yeast for tolerance to stresses encountered during fermentation of lignocellulosic residues to produce bioethanol. Microb. Cell Fact. 13:47. doi: 10.1186/14752859-13-47

Conflict of Interest: The authors declare that the research was conducted in the absence of any commercial or financial relationships that could be construed as a potential conflict of interest.

Publisher's Note: All claims expressed in this article are solely those of the authors and do not necessarily represent those of their affiliated organizations, or those of the publisher, the editors and the reviewers. Any product that may be evaluated in this article, or claim that may be made by its manufacturer, is not guaranteed or endorsed by the publisher.

Copyright (c) 2021 Cagnin, Gronchi, Basaglia, Favaro and Casella. This is an openaccess article distributed under the terms of the Creative Commons Attribution License (CC BY). The use, distribution or reproduction in other forums is permitted, provided the original author(s) and the copyright owner(s) are credited and that the original publication in this journal is cited, in accordance with accepted academic practice. No use, distribution or reproduction is permitted which does not comply with these terms. 